\title{
A Survey and Error Analysis of the Bă-Sentence Based On HSK Dynamic Composition Corpus
}

\author{
Yan LIU, Shan WU \\ College of Humanities \& Law, North China Univ. of Tech., Beijing, China (jljaly@ 163.com)
}

\begin{abstract}
This paper analyzes erroneous Bă-Sentences which are collected from the HSK dynamic composition corpus. Two hundred erroneous Bă-sentences are divided into five categories according to their error types. After examining the cause of the biased errors,the paper has put forward some suggestions on the strategies that can be employed in teaching the Br̆-sentence.
\end{abstract}

Keywords—Bă-Sentence, biased errors, teaching suggestions

\section{基于 HSK 动态作文语料库的“把”字句偏误分析 \\ 刘妍巫珊 \\ 北方工业大学文法学院, 北京, 中国}

摘 要 本文对 HSK 动态作文语料库中 “把” 字句的偏误进行分析, 归纳总结留学生在学习和使用 “把” 字句时出现的五类偏误 问题，运用中介语理论和偏误分析理论探究导致这些偏误的原因，并给出相应的教学建议。

关键词 “把” 字句；偏误；教学建议

\section{1. “把”字句的语义}

“把” 字句是指在谓语动词前头用介词 “把” 引出受 事、对受事加以处置的一种主动句, 因此, “把” 字句又叫 处置式。“把” 字句的语义特点, 从 “宾语提前说”、“处置 说”，后来发展成为 “宽泛的处置说” 并包括 “致使说” 在 内。随着研究的深入, 不断有学者专家提出更为细致贴切 的定义。目前, 我们普遍认同 “把” 字句是处置式这一观 点, 所谓处置, 就是谓语中的动词所表示的动作对把字引 出的受事施加影响, 使它产生某种结果, 发生某种变化, 或处于某种状态。第一, “把” 字句中的谓语动词一般不能 单独出现, 尤其不能出现单音节动词。通常后面有补语、 宾语、动态助词或动词重叠式, 动词后的成分一般表示动 作已经完成。第二, “把” 字句中的宾语一般是在意念上有 定的、已知的人或事物, 前面常带有 “这、那” 等修饰语。 第三, 谓语动词是对受事施加影响, 一般具有动作性的及 物动词, 所以不及物动词、判断动词、趋向动词、心理动 词等不能作为谓语动词。

\section{2. 留学生的偏误分析}

本文的语料来源于北京语言大学 “HSK 动态作文语料 库”，在错句检索中检索 “把” 字句，发现 “把” 字句的偏 误一共有 585 条, 从中随机抽取 200 条作为研究样本, 发 现留学生产生的偏误一共有五类, 分别是: 回避、泛化、 误用、遗漏、乱序。对这五类问题进行分析, 统计得出偏 误率, 能直观地看出留学生在使用 “把” 字句时的主要错 误。

\section{1 偏误类别}

\section{1.1 回避}

回避是指在句法、语义和语用等方面符合 “把” 字句 的要求却没有采用把字结构而产生的偏误。据统计, 这类 偏误一共有 76 例, 占整个偏误类型的 $38 \%$ 。举例:

* (1) 他对我要求特别高, 因为我是独生子, 所以他 的全部希望寄托在我身上。

*（2）我想这个庙搬到山下怎么样?

这类句子符合把字结构的语义要求, 只要加上把字再 把句子稍微调整就是正确的 “把” 字句。这类偏误是留学 
生误用 “把” 字句的主要类型, 体现了留学生对 “把” 字 句的掌握不准确, 概念模糊, 因此采取回避的策略。

\section{1.2 泛化}

泛化与回避相反, 是指在语义、语用及语境等方面不 需要使用 “把” 字句却用了把字结构而产生的偏误类型。 这类偏误一共有 38 例, 占总偏误类型的 $28.5 \%$ 。

* (3) 但是经过自己独立地反复地思考, 把我慢慢地 变成了一个能够独立判断的人。

* (4) 故事中把三个和尚之间发生的问题和那个结果 给我们表示的意思是这样的。

* (5) 把舌头太卷的话, 没有声音, 反倒把舌头卷松 一些的话, 像哑巴想尿的声音似的, 怎么办?

这 3 句直接去掉 “把” 字, 就能变成一个正确的句子。 2.1. 3 误用

误用是指在语义和语用方面有使用 “把” 字句的 要求, 但是在构成把字结构时出现的搭配失误。这类偏误 有三类, 共有 44 例, 占偏误类型的 $22 \%$, 分别是谓语动词 误用、宾语误用、补语误用。

*（6）一般的年轻人如果在家里发生这样的问题, 有 可能把这样的问题在外面泄气, 这样的话, 他跟他的父母 之间的代沟永远不可解决。

* (7) 我认为尤其是来华留学生比别的情况的人把这 个 “滋味” 了解得更深一些, 我也是来华留学生, 曾经尝 过好几次这种 “滋味”, 到此也几乎每日都尝。

*（8）我会思考犯的过错, 不会是唉声叹气, 怨天尤 人, 不会变得一蹶不振, 而是要双倍的努力, 把以往丰收 的经验思考, 从零开始。

这 3 句是误用偏误中的谓语动词误用, 在误用偏误中 有 31 例, 占有很高的比例, 也说明了留学生在学习 “把” 字句时对于谓语动词的掌握不足。其中, (6) 句中的 “泄 气” 是名词, 在 “把” 字句中不能用作谓语, 应该改成 “发 泄”。(7) 句中 “了解” 属于感受类的心理动词, 不具有动 作性, 所以不能用作 “把” 字句的谓语动词。(8) 句中 “思 考”与 “经验” 搭配不当, 应该改成 “总结”。

*（9）他决心把我们的语文能力提高。

* (10) 如果我奶奶来荷兰, 她总是把几本汉语课本给 我带来。那个时候学习写汉字不过是一个爱好, 所以我并 不努力。

这 2 句是宾语误用, 这类偏误共有 3 例。其中, (9) 句中 “能力” 不能与 “提高” 并用, 应该改成 “水平”。(10) 句中宾语 “几本汉语课本” 不是已知的有定的事物, 所以 应该加上修饰词 “这、那”。

*(11) 他把行李掏了半天。

* (12) 我把我的声调怎么改也改不了了。
这 2 句是补语误用, 这类偏误共有 10 例。其中, (11) 句中的 “半天” 表示时间的延长, 不表示状态, 与 “把” 字句的语义不符。(12) 句 “改也改不了了” 是可能补语, 表示动作的结果、趋向可能不可能实现, 不含处置义, 因 此不能用作 “把” 字句。

\section{1.4 遗漏}

遗漏是指句中的某一句子成分缺失导致的语句偏误, 主要是谓语动词的遗漏或谓语动词后的成分遗漏。这类偏 误一共有 28 例, 占整个偏误的 $14 \%$ 。

* (13) 为了更方便, 他们商量怎么样能把这些工作更 轻松一些。

* (14) 我决定了, 一定要考上大学, 为了报答他的关 心, 也为了我的未来, 那天开始我把书朋友, 很拼命地学 习。

谓语动词的遗漏主要是留学生对于 “把” 字句的结构 “S + 把 $+\mathrm{N}+\mathrm{V}+$ 其他” 不太了解而导致的偏误, 这些句子符合 “把” 字句的使用要求, 只要添上合适的谓语动词就能成 为一个正确的句子。这类偏误一共有 8 例。

*（15）他们开玩笑地把火柴吹, 不让新娘顺利地点烟。

*（16）他也来不及和老师的弟弟自我介绍等, 先用英 语、汉语把病情介绍, 转达意思。

这 2 句是谓语动词后的成分遗漏导致的偏误, 导致偏 误的原因是不符合 “把” 字句的语义 “把” 字句中的谓语 动词一般不能单独出现, 尤其不能出现单音节动词。通常 后面有补语、宾语、动态助词或动词重叠式” 的要求, 这 类偏误出现较多, 共有 20 例。

2. 1.5 乱序

乱序是句子成分的颠倒而导致的语序上的错误。这类 偏误共有 13 例, 占 $6.5 \%$ 。

*（17）但是要生活下去的话把应该做的事情自己做完 全。

*（18）别的和尚问他 “秘诀是什么” , 他把这个和尚 告诉了以前的事。

这类偏误主要是把字结构中出现的成分颠倒, 包括谓 语动词与名词之间的乱序, 名词和宾语之间的乱序, 只要 把相应的句子成分对调, 就是正确的句子。

\section{2 小结}

从以上偏误类别中可以看出, 回避类偏误占的比重最大, 其次是泛化, 这两类偏误一个是该用而未用 “把” 字句, 一个是不该用却用了 “把” 字句, 从中可以看出留学生在 学习和使用 “把” 字句时对 “把” 字句的概念模糊不清, 没有掌握好 “把” 字句的处置义, 以及对于 “把” 字句的 结构 “ $\mathrm{S}+$ 把 $+\mathrm{N}+\mathrm{V}+$ 其他” 存在误区, 在用与不用 “把” 字句 
时难以定夺。

\section{3. 导致偏误的原因}

造成这些偏误的原因有两大类。从客观方面来看, 主要是 由语言系统和文化背景差异造成的, 包括学习者母语的影 响, 目的语的影响以及文化差异。从主观方面来看, 留学 生学习汉语的时间长短、学习方法和策略以及汉语教师的 教学等都会对留学生使用汉语产生影响。

\section{1 母语负迁移}

在外语的学习过程中, 学习者对母语的使用会对外语 的学习产生影响, 由于母语中某些与外语相异的成分导致 对外语的掌握造成不良影响叫做母语负迁移。最常见的是 词法和句法的负迁移。母语负迁移虽然会对学习者在学习 外语时造成一些影响, 但是在学习外语的初级阶段, 也不 失为一个帮助学习者快速理解目的语的方法, 只要在学习 过程中加以指导, 也能把母语负迁移变成母语正迁移。

\section{2 目的语的影响}

学生由于掌握目的语知识的不足, 把他所学的不充分 的、有限的目的语知识, 套用在新的语言现象上, 结果产 生偏误。这种偏误在心理学上叫做 “过度泛化”。在 “把” 字句的偏误中, 回避类偏误就是留学生对于 “把” 字句的 掌握不足，不知道该不该用继而不使用 “把” 字句造成的 偏误。

* (19) 长辈们有这种想法是因为他们用他们的生活态 度以及价值观强加于晚辈的身上, 他们常常用一句老话去 教那些年轻人: “我走的桥比你们走的路还多”。

这个错句是该用介词 “把” 却误用了介词 “用”, 虽然 “用” 作为介词的时候可以表示 “拿、以”, 句义上说得通, 但是 “用” 并没有表示处置义, 不具有动作性, 因此不能 与后面的 “强加于” 搭配。这就是留学生在没有很好地掌 握 “把” 字句的情况下受目的语的影响不会使用正确的句 式, 从而用其他熟悉的词语或句式来代替导致的偏误。

\section{3 文化差异}

不同的国家有各自的文化风俗、语言习惯，这就造成 了同一个意象在不同的国家有不同的表达和解释, 从而导 致了语言学习者在学习和使用外语时对于意象的表达会有 偏误。比如中国和韩国的词汇在意义表达上就有区别。这 些词汇方面的差异直接导致留学生在使用汉语时词义表达 不准确, 造成语义上的歧义, 也就导致了遗漏和误用类的 偏误。

\section{4 学习时间不够}

汉语水平的高低很大程度上取决于学习汉语的时间长 短, 从不同留学生造成的偏误来看, 水平高的留学生在使 用汉语时所犯的偏误数量比较少, 并且偏误的类型也少, 在 “把” 字句的使用过程中, 高级 (A 级) 留学生所犯的 偏误以回避和误用为主, 中级 (B 级) 留学生也是以回避 和误用为主, 但是也有遗漏和泛化, 初级 ( C 级) 留学生 则是以回避、泛化和遗漏为主, 兼有误用和乱序。这也反 映了留学生在学习 “把” 字句的过程中从简单到复杂, 由 词法到句法循序渐进。

\section{5 学习方法和策略}

每一种语言都有一定的学习方法和空门, 有一定的学 习顺序, 只要掌握了正确的学习方法, 那么外语的学习过 程就会变得简单顺利。比如学习汉语, 首先得学会拼音, 然后把拼音和汉字对上号, 最后理解汉字的意思也就是词 义, 最后学习语法。同样, 学习英语得先学习音标, 然后 会拼写, 最后了解词义, 之后按照语法造句。这是学习外 语的基本过程, 但是每个人有不同的学习策略, 会对习得 效果产生不同的影响。

\section{4. 教学建议}

通过对 “HSK 动态作文语料库” 中的语料进行分析可 以看出, 在使用 “把” 字句的过程中, 留学生产生的偏误 问题主要是回避类和泛化类。究其根本, 还是留学生对于 “把” 字句的语义理解模糊, 对 “把” 字句的句式结构掌 握不足。因此, 针对以上两点, 给出以下建议:

（一）针对留学生对 “把” 字句的语义理解模糊, 教师 可以采用抽丝剥茧的方法, 先从整体上解释 “把” 字句的 处置义, 从宏观层面阐述处置义的意思, 也就是谓语中的 动词所表示的动作对把字引出的受事施加影响, 使它产生 某种结果, 发生某种变化, 或处于某种状态, 让留学生对 于 “把” 字句的使用场合有个大概的定义。接着再从 “把” 字句中的词法入手, 谓语动词的限制即一般不能单独出现, 尤其不能出现单音节动词, 通常后面有补语、宾语、动态 助词或动词重叠式。宾语的有定即宾语一般是在意念上有 定的、已知的人或事物, 前面常带有 “这、那” 等修饰语。 这样可以大大地减少留学生在使用 “把” 字句时导致的遗 漏类偏误。

（二）针对留学生对 “把” 字句的句式结构掌握不足, 教师可以采用循序渐进的教学策略, 遵循由易到难、由简 到繁、由具体到抽象的原则。在教学中, 可以从最简单最 基本的 “把” 字句开始, 由简到繁、由易到难, 有利于学 
生的理解。

(三) 针对不同水平的留学生导致的偏误问题不同, 教 师可以采用分层教学的方法来教授 “把” 字句。所谓分层 教学就是教师根据学生现有的知识、能力不同进行分组教 学, 针对每一阶段、每一水平的学生, 教授不同的知识, 做到因材施教。正如以上得出的不同水平的留学生的偏误 比较, 不同水平的留学生所犯的偏误不同, 可以根据不同 留学生主要导致的偏误进行相应的教学改进。初级 (C 级) 留学生掌握的汉语知识较少, 汉语水平较低, 因此可以先 从简单的把字句开始教学, 同时辅以字词的讲解, 帮助初 级水平的留学生夯实基础, 减少留学生的遗漏偏误; 中级 (B 级) 留学生已经掌握了一定的汉语词汇和句式, 此时 可以从把字结构入手, 对把字句的语义和语用详细剖析, 纠正留学生的误用和泛化偏误; 高级 (A 级) 留学生的汉 语水平相对较高, 因此可以进行词汇和句式的对比教学, 帮助留学生区分相近词汇, 区别不同句式的使用场合, 有 效地减少留学生的回避和误用类偏误。

(四) 注重实践, 加强语境教学, 通过对语境的模拟使 留学生有个良好的语言环境进行交流。教师在对把字句的 语义和语用进行讲解的同时, 应该辅以把字句的练习, 把 理论和实践相结合。而句子是个孤立的个体, 只有每个句 子串联起来, 才能形成完整的语篇。所以, 对于把字句的
教学, 教师应该放到具体的语境中, 设置不同的语境, 如 口头语境、书面语境等, 加强留学生对于把字句的处置义 的理解和感受, 形成对把字句的宏观把握。

Supported by Beijing Higher Education Young Elite Teacher Project YETP1432

\section{参考文献(References)}

[1] Ellis. R, Second Language Acquisition. Oxford: Oxford University Press, 1997.

[2] Cheung, Hung-nins, A comparative study in Chinese grammars the ba-construction. Journal of Chinese Linguistics, 1973 (1).

[3] Li, C, Thompson, S. Mandarin Chinese: A Functional Reference Grammar, CA: University of California Press, 1981.

[4] S. P. Corder, The Significance of Learner's Errors. Penguin: International Review of Applied Linguistics, 1967, (4).

[5] S. P. Corder, Introducing Applied Linguistics. Harmondsworth: Penguin Books Ltd, 1973.

[6] Zhang Baolin. Avoidance and Overgeneralization-An Investigation of Acquisition of the Bă-Sentence Based on the HSK Dynamic Composition Corpus. Chinese Teaching in the World, 2010 (04). 